\title{
Improvement of Scanning Tunneling Microscopy Resolution with H-Sensitized Tips
}

\author{
J. I. Martínez, ${ }^{1, *}$ E. Abad, ${ }^{1}$ C. González, ${ }^{1,2}$ F. Flores, ${ }^{1}$ and J. Ortega ${ }^{1}$ \\ ${ }^{1}$ Departamento de Física Teórica de la Materia Condensada, Universidad Autónoma de Madrid, ES-28049 Madrid, Spain \\ ${ }^{2}$ Departamento de Superficies y Recubrimientos, Instituto de Ciencia de Materiales de Madrid (CSIC), ES-28049 Madrid, Spain
}

(Received 9 February 2012; published 13 June 2012)

\begin{abstract}
Recent scanning tunneling hydrogen microscopy (STHM) experiments on PTCDA (perylene-3,4,9,10tetracarboxylic-3,4,9,10-dianhydride)/ $\mathrm{Au}(111)$ have shown unprecedented intramolecular and intermolecular spatial resolution. The origin of this resolution is studied using an accurate STHM theoretical simulation technique that includes a detailed description of the electronic structure of both the tip and sample. Our results show that $\mathrm{H}_{2}$ molecules are dissociated on the Au tip; the adsorbed $\mathrm{H}$ atoms change the density of states at the Fermi level $\left(E_{F}\right)$ of the tip, increasing its $p$-orbital character and reducing the $s$-orbital contribution. Also, due to the interaction with the H-decorated tip, $E_{F}$ is shifted to the middle of the PTCDA lowest unoccupied molecular orbital peak, increasing dramatically the density of states of the sample at $E_{F}$. These effects give rise to the enhanced STHM resolution.
\end{abstract}

PACS numbers: 68.37.Ef, 71.15.Mb, 73.20. $-\mathrm{r}, 73.40 .-\mathrm{c}$

Three decades ago, the invention of scanning tunneling microscopy (STM) [1] revolutionized the surface science community allowing us, for the first time, to obtain atomic resolution by probe microscopy. Combined with atomic force [2] and kelvin probe force microscopy techniques [3], STM is widely used to characterize electronic structure and corrugation properties of semiconducting surfaces and interfaces at the nanoscale level [4]. Although nowadays STM is a standard technique for surface characterization, many aspects of its operation are not well understood [4-7].

In the recently developed scanning tunneling hydrogen microscopy (STHM) [7-9], improved geometrical resolution is achieved by filling (flooding) the STM chamber at low temperature with molecular hydrogen [7]. A paradigmatic case for STHM is PTCDA (perylene-3,4,9,10-tetracarboxylic-3,4,9,10-dianhydride)/Au(111) [8,9], where a spectacular enhancement of the molecule intramolecular resolution has been obtained; moreover, the intermolecular hydrogen bonds for the herringbone PTCDA monolayer on $\mathrm{Au}(111)$ are observed in the STHM image [8]. Although $\mathrm{H}_{2}$ molecules seem to dissociate on gold clusters and other nanostructures [10-14], it has been argued that the presence of $\mathrm{H}_{2}$ molecules between the tip apex and the sample is the key element for the improved resolution [7,9]; however, the way in which $\mathrm{H}_{2}$ molecules at the STM junction affect the STM image remains a mystery.

The lack of understanding of the physical mechanism underlying STHM resolution is largely related to our lack of theoretical simulations for STHM images, which is a very challenging task [4]. In the standard application of the Tersoff-Hamman approach [15], the STM tunneling current is simply proportional to the density of states (DOS) at the Fermi level $\left(E_{F}\right)$ of the sample at the position of the tip; thus, contour maps of this DOS have been used to generate theoretical STM images. This method has proven very useful, obtaining qualitatively correct STM images for moderately simple interfaces. Nevertheless, this theoretical approach fails for slightly more complex systems, for instance, when $p$ (or $d$ ) orbitals in the tip play a significant role in the STM current [6], or in the case of tip contamination.

In this work, we analyze theoretically the physical mechanism of STHM resolution for PTCDA/Au(111) $[8,9]$. For this purpose, we use an accurate STM theoretical simulation technique that includes a detailed description of the electronic properties of both the tip and the sample. Using this technique, based on a combination of a Keldysh Green's function formalism and local orbital density functional theory (DFT) [16], we analyze the effect on the STM images of both atomic and molecular hydrogen interaction with the tip and sample. Our results show that the enhanced spatial resolution observed in STHM is due to atomic $\mathrm{H}$ adsorbed on the metallic tip. This conclusion is reinforced by the observation that, in our calculations, the $\mathrm{H}_{2}$ molecules dissociate on the Au tip, as previously reported for other Au nanostructures [10-14]. Our theoretical analysis reveals the physical mechanism underlying STHM spatial resolution. First, the interaction with the $\mathrm{H}$-decorated tip shifts the position of $E_{F}$ to the middle of the narrow PTCDA lowest unoccupied molecular orbital (LUMO) peak, magnifying the STM current for very low bias voltages $(\sim 5-10 \mathrm{mV})$; moreover, the adsorption of atomic $\mathrm{H}$ on the tip changes the orbital character of the tip DOS at $E_{F}$, increasing the DOS for the $p$ orbitals while reducing it for the $s$ orbitals. The combination of these two factors results in an improved resolution on the STM images, including the experimentally observed enhanced visibility of the PTCDA intermolecular bonds [8].

In order to simulate theoretically the STHM images, we split the system into sample and tip, where the sample is the herringbone PTCDA/Au(111) surface and the effect of hydrogen is analyzed by adding hydrogen atoms (or 
molecules) to the tip in different configurations. In our calculations we have explored different $\mathrm{Au}, \mathrm{W}$, and mixed $\mathrm{Au} / \mathrm{W}$ tips (see Supplemental Material [17]) with similar results. In this paper we focus on the Au case.

In the tunneling regime at low temperature, the STM current is given by [16]

$$
I=\frac{4 \pi e^{2}}{\hbar} \int_{E_{F}}^{E_{F}+e V_{s}} d \omega \operatorname{Tr}\left[\hat{T}_{t s} \hat{\rho}_{s s}(\omega) \hat{T}_{s t} \hat{\rho}_{t t}(\omega-e V)\right]
$$

where $V_{s}$ is the surface voltage, $\hat{\rho}_{t t}(\omega)$ and $\hat{\rho}_{s s}(\omega)$ are the DOS matrices, in the local orbital basis, associated with the tip and sample, while $\hat{T}_{t s}$ and $\hat{T}_{s t}$ are the local orbital Hamiltonian matrices coupling tip and sample (see Ref. [16] for details). In our STM simulations, $\hat{\rho}_{s s}(\omega)$ is calculated using the Hamiltonian for PTCDA/Au(111) obtained with the local orbital DFT code [18], where charging energy effects are taken into account to properly determine the molecule transport gap $[19,20]$ (see Ref. [21] for details). This is a critical correction to obtain reliable STM images, since these images depend sensitively on the value of this transport gap and, in particular, on the position of $E_{F}$. The adsorption distance for PTCDA on $\mathrm{Au}(111)$ is taken from the experimental evidence (3.4 $\AA$ [22]). $\hat{\rho}_{t t}(\omega)$, as well as the different hopping matrix elements between orbitals in the tip and sample (defining the coupling terms $\hat{T}_{t s}$ and $\hat{T}_{s t}$ ) are also calculated using local orbital DFT [16,18].

Figure 1A.t shows the theoretical STM image for the herringbone PTCDA/Au(111) surface, for the case of a clean Au tip. In order to mimic the experimental conditions $[8,9]$, we have used a bias voltage of $\mathrm{V}_{s}=230 \mathrm{mV}$; the scanning height is $4 \AA$ (a very similar image is obtained for $3.5 \AA$ or $4.5 \AA$ ). In the PTCDA/Au(111) surface, the LUMO is located approximately $0.5 \mathrm{eV}$ above $E_{F}$ [21]; the interaction with the Au tip shifts $E_{F} 0.27 \mathrm{eV}$ closer to the PTCDA LUMO peak, as shown in Fig. 3A, so that a bias voltage of approximately $0.2 \mathrm{~V}$ is enough to tunnel to this state. Figure 1A.e shows the corresponding experimental STM image [8]. The agreement between both images is excellent.

At this stage (H-free Au tip), the STM image does not reveal any significant information about the PTCDA intramolecular structure or the intermolecular bonding between neighboring molecules. In order to analyze the improved resolution when hydrogen is added to the STM chamber, we have first explored the inclusion of a $\mathrm{H}_{2}$ molecule between the tip and sample [9] for different geometries, and either adsorbed on the tip or on the PTCDA molecule. As a representative example, Fig. 1B.t1 shows the theoretical STM image for the case of a $\mathrm{H}_{2}$ molecule adsorbed with the molecular axis along the vertical direction $(z)$ between the tip and sample. The tunneling current associated with the $\mathrm{H}$ orbitals yields an STM image that reflects the PTCDA LUMO density [Fig. 1B.t2]; to this, we have to add the STM current related to the Au orbitals (and interference terms) in order to obtain the total STM image,

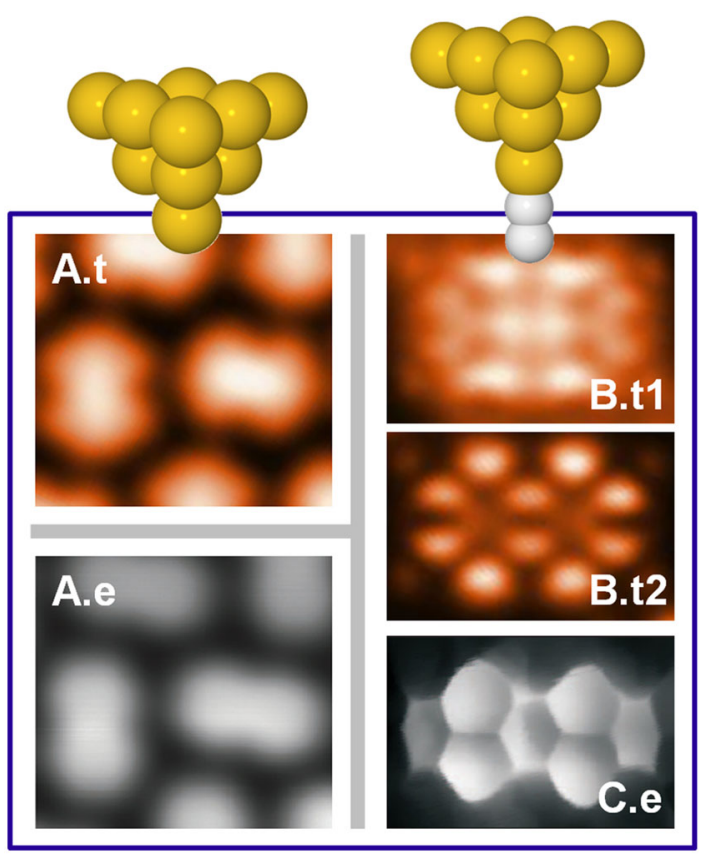

FIG. 1 (color online). Comparison of experimental [9] and theoretical STM images for PTCDA on $\operatorname{Au}(111)$ : (A) Theoretical (t) and experimental (e) STM image for the clean Au tip. (B) $d I / d V$ images at $V_{s}=0$ for a Au tip with a $\mathrm{H}_{2}$ molecule adsorbed between tip and sample: (t.1) total; (t.2) $\mathrm{H}_{2}$ contribution. (C.e) Experimental STHM image. Top: schematic representation of the last few atoms for the tips used in the theoretical images. Scanning height: (A.t): $3.8 \AA$; (B.t1 and B.t2): $4.5 \AA$.

Fig. 1B.t1. Although this theoretical STM image presents significant intramolecular resolution, it does not resemble the experimental STHM image, Fig. 1C.e. Similar results have been obtained for other Au tip $+\mathrm{H}_{2}$ geometries, e.g. see Supplemental Material [17]. We conclude that the presence of $\mathrm{H}_{2}$ molecules between the tip and sample cannot explain the observed STHM image.

The adsorption geometry for this case was obtained through a constrained minimization in which the $\mathrm{H}$ atoms are allowed only to relax along the $z$-direction. In free relaxations we find that the $\mathrm{H}_{2}$ molecules are spontaneously dissociated, resulting in atomic $\mathrm{H}$ atoms adsorbed on the Au tip (see videos in Supplemental Material [17]). Thus, we have explored STM images for different $\mathrm{Au}$ tips decorated with $\mathrm{H}$ atoms, where the tip geometry is calculated relaxing the atomic positions. Figure 2C.t1 shows the theoretical STM that best resembles the experimental STHM image (Fig. 1C.e, [9]); this image corresponds to a Au tip with an atomic $\mathrm{H}$ adsorbed on the apex and all nearby bridge positions saturated with $\mathrm{H}$ atoms (see inset in Fig. 3A). The bottom panel of Fig. 2 shows the normalized $d I / d V$ along different high symmetry scanning lines for the 2C.t1 image. Figure 2C.t3 shows this image in a larger scale, showing how the improved resolution is collaterally manifested through an enhancement of the 


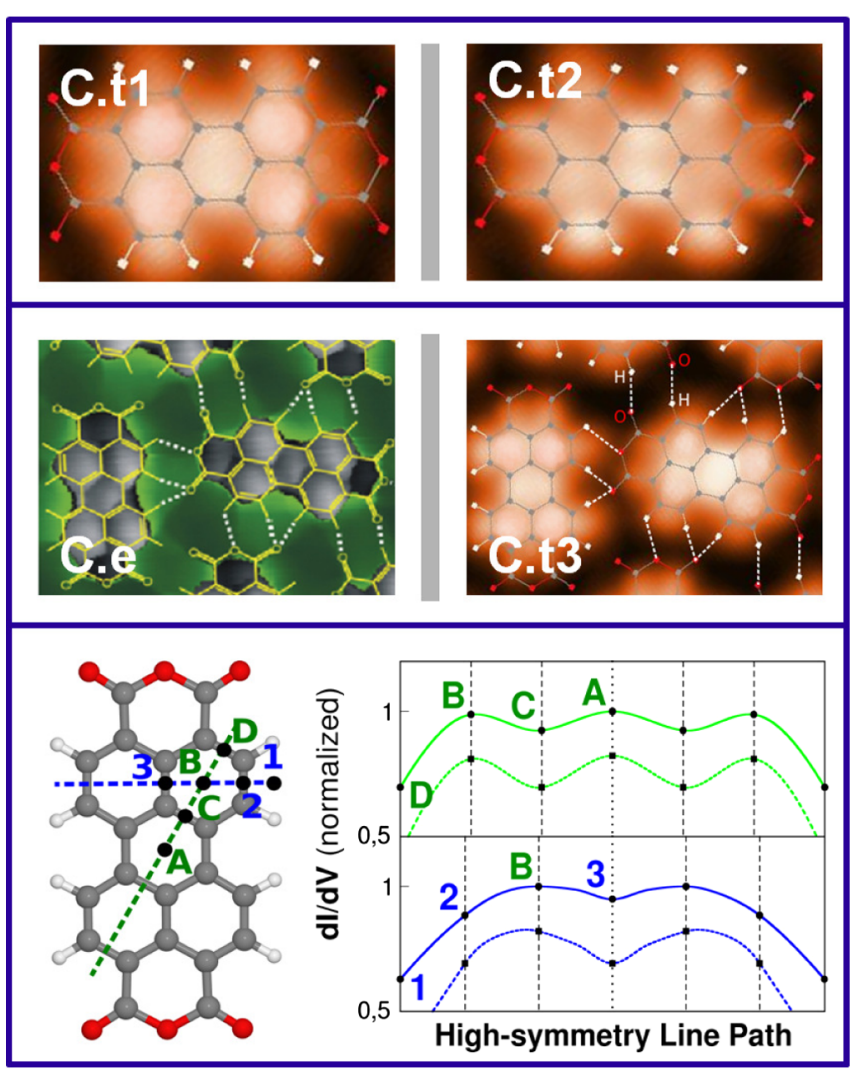

FIG. 2 (color online). $d I / d V$ images at $V_{s}=0$ for the herringbone monolayer of PTCDA molecules on $\mathrm{Au}(111)$. Scanning height: (C.t1, C.t3): $3.8 \AA$; (C.t2): 3.5 A. (C.e) Experimental STHM image [8]. Bottom panel shows the normalized $d I / d V$ along different high-symmetry scanning lines (see bottom left) for the C.t1 image (solid line), and the Au apex (see Fig. 3) contribution (dashed line).

visibility of the intermolecular bonding between neighboring PTCDA molecules, as observed experimentally (see Fig. 2C.e, [8]); in particular, the intermolecular bonds between $\mathrm{O}$ and $\mathrm{H}$ atoms in neighboring PTCDA molecules are quite visible. Figure $2 \mathrm{C}$.t 1 corresponds to a tip sample distance of $3.8 \AA$; for a distance of $4.5 \AA$, the image is similar, but slightly blurred, while for $3.5 \AA$ the image starts to resemble the LUMO electron density (see Fig. 2C.t2). This reveals that the experimental STHM image [Fig. 1C.e] is essentially a blurred image of the PTCDA LUMO state. Our simulations suggest that the case shown in Fig. 2C.t2 cannot be observed experimentally, since for a tip sample distance of $3.5 \AA$, the $\mathrm{H}$ atom on the tip apex is no longer stable.

Our analysis reveals that the resolution enhancement observed in STHM is related to the dissociation of $\mathrm{H}_{2}$ molecules, resulting in atomic $\mathrm{H}$ adsorbed on the tip. We further analyze the physical mechanism for this improved resolution. Figure $3 \mathrm{~A}$ shows the DOS on the PTCDA monolayer and how the interaction between the tip and surface shifts the position of the Fermi level with respect to the PTCDA DOS: in the case of the clean Au tip, $E_{F}$ moves
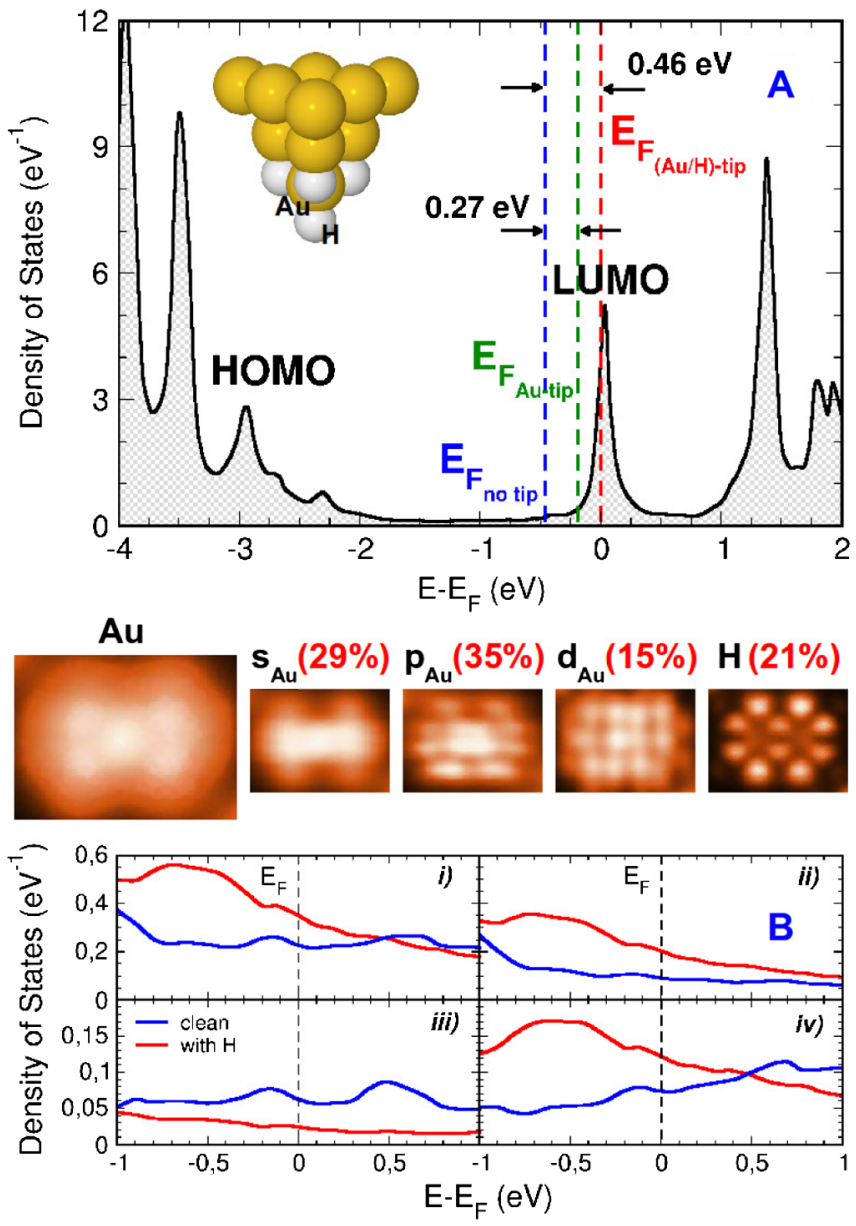

FIG. 3 (color online). (A) DOS on the PTCDA monolayer. The interaction with the Au tip shifts $E_{F}$ upwards with respect to the molecular DOS; for the H-decorated Au tip (shown in the inset) $E_{F}$ is in the middle of the narrow LUMO peak. Middle: different contributions to the STM image of Fig. 1B.t1 coming from the Au apex (also separated into $s, p$ and $d$ contributions) and the apex $\mathrm{H}$ atom. (B) DOS on the $\mathrm{Au}$ apex atom around $E_{F}$ for the clean $\left(E_{F_{\text {no tip }}}\right)$ and H-decorated $\left(E_{F_{(\mathrm{Au} / \mathrm{H})-\text { tip }}}\right)$ Au tips: (i) total; (ii) $d$-orbitals; (iii) $s$-orbitals; (iv) $p$-orbitals.

$0.27 \mathrm{eV}$ upwards, closer to the LUMO peak, while for the $\mathrm{Au}$ tip decorated with $\mathrm{H}$ atoms (inset in Fig. 3A) the Fermi level is pinned at the LUMO peak. Due to this shift, the DOS of the sample, $\hat{\rho}_{s s}$ (see Eq. (1)) at $E_{F}$ is much larger, increasing substantially the conductance for very low voltages. This is in agreement with the experimental observation that the clearest geometric resolution is obtained in the limit of zero bias [7]. The position of $E_{F}$ in the middle of this narrow peak is also reflected in the peak observed experimentally at zero bias in the $d I / d V$ spectrum [9].

The middle panel of Fig. 3 shows the different contributions to the STM image of Fig. 2C.t1. The s-orbital image is similar to the clean tip case [Fig. 1A.t]; now, however, this contribution is substantially reduced: the interaction with the adsorbed $\mathrm{H}$ atoms changes the orbital character of the DOS close to $E_{F}$ for the Au apex atom, as 


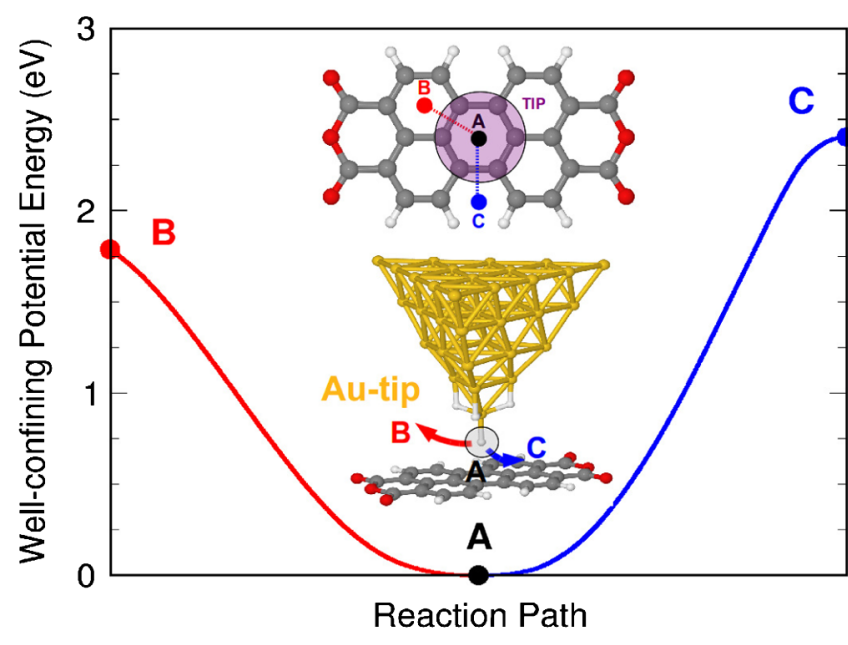

FIG. 4 (color online). Potential energy surface for the lateral displacement of the $\mathrm{H}$ atom at the tip apex.

shown in Figs. 3B.i-iv, significantly reducing the $\mathrm{Au}$ $s$-orbital DOS while increasing the DOS for the directional $p$ and $d$ Au orbitals. Thus, we find that the enhanced resolution is mainly due to the $p$-orbital contribution (the contribution from $d$-orbitals is less important due to their shorter range), with some contribution also from the $\mathrm{H}$ atom at the apex. This finding is in agreement with a recent study using CO-functionalized tips [6], where it was shown that the intramolecular resolution in the STM images was due to $p$-orbitals tip states.

Our results show that, under a $\mathrm{H}_{2}$-saturated atmosphere, some $\mathrm{H}_{2}$ molecules dissociate on the tip, the resulting $\mathrm{H}$ atoms adsorbing on bridge positions and on the tip apex. This is confirmed by total energy plane wave DFT [23] calculations that show that the H-contaminated Au tip (inset of Fig. 3A) is $0.42 \mathrm{eV}$ lower in energy than the clean Au tip plus two $\mathrm{H}_{2}$ molecules [24]. We have also analyzed the stability of this H-decorated Au tip. For this purpose, we have performed different calculations where only one PTCDA molecule and the tip are involved. In these new calculations, the tip is fixed over one hollow position on the PTCDA molecule (see Fig. 4). Then, we have performed constrained relaxations, fixing the $x, y$ coordinates of the apex $\mathrm{H}$ atom, while its vertical coordinate $z$ and the rest of the system are relaxed until it reaches the energy minimum. Doing this for different $x, y$ positions we calculate the potential energy surface (PES) for the motion of the apex $\mathrm{H}$ atom. Figure 4 shows the result for two different paths, $A-B$ and $A-C$ (see inset), as calculated with a DFT plane wave code PWSCF [23] (similar results are obtained using the local-orbital code FIREBALL [18]). These calculations show that the position of this $\mathrm{H}$ atom in between the tip and the PTCDA molecule, directly below the tip apex, is very stable: the energy barrier for this $\mathrm{H}$ atom to leave its potential well is of the order of $2 \mathrm{eV}$. Thus, it can be assumed that the $\mathrm{H}$ atom stays below the tip during the complete STM measurement, following the apex every time the tip is moved (see video in Supplemental Material [17]). Other starting positions for the tip have been checked obtaining very similar results.

The results of Fig. 4 can be used to understand the origin of the inelastic features observed in STHM; whereby, the STHM enhanced resolution disappears above a voltage $V_{\text {inel }}$ of the order of $50 \mathrm{mV}$ [9]. Using a harmonic approximation for the PES in Fig. 4, we estimate a vibrational excitation energy of approximately $42 \mathrm{meV}$ for deuterium. This result suggests that inelastic electron tunneling induces a stepwise excitation of higher vibrational modes $[26,27]$, resulting in a loss of resolution in the STM image.

In conclusion, we have analyzed the origin of the intramolecular and intermolecular resolution attained in STHM for the paradigmatic PTCDA/Au(111) case. We find that $\mathrm{H}_{2}$ molecules are easily dissociated by the Au tip, resulting in atomic $\mathrm{H}$ adsorbed close to the tip apex. The interaction of the H-decorated Au tip with PTCDA/Au(111) pins $E_{F}$ in the middle of the narrow PTCDA LUMO peak, increasing dramatically the DOS of the sample at $E_{F}$ and allowing electrons to tunnel from or to this peak at very low bias voltages. Also, the adsorbed $\mathrm{H}$ atoms change the DOS of the Au tip at $E_{F}$, increasing its $p$-wave character and reducing the $s$-orbital contribution. The combination of these two effects gives rise to the enhanced STHM intramolecular resolution, as well as the increased visibility of the intermolecular bonds.

This work is supported by Spanish MICIIN under Contract No. FIS2010-16046, the CAM under Contract No. S2009/MAT-1467, and the European Project MINOTOR (Grant No. FP7-NMP-228424). J. I. M. acknowledges funding from Spanish MICINN through Juan de la Cierva Program, E. A. the financial support by Consejería de Educación de la CAM, FSE, and European Project MINOTOR, and C. G. the CSIC JAE.

*joseignacio.martinez@uam.es

[1] G. Binnig, H. Rohrer, C. Gerber, and E. Weibel, Appl. Phys. Lett. 40, 178 (1982).

[2] G. Binnig, C. F. Quate, and C. Gerber, Phys. Rev. Lett. 56, 930 (1986).

[3] M. Nonnenmacher, M.P. O'Boyle, and H. K. Wickramasinghe, Appl. Phys. Lett. 58, 2921 (1991).

[4] L. Gross, Nature Chem. 3, 273 (2011).

[5] P. Jelinek, M. Svec, P. Pou, R. Pérez, and V. Cháb, Phys. Rev. Lett. 101, 176101 (2008).

[6] L. Gross, N. Moll, F. Mohn, A. Curioni, G. Meyer, F. Hanke, and M. Persson, Phys. Rev. Lett. 107, 086101 (2011).

[7] R. Temirov, S. Soubatch, O. Neucheva, A. C. Lassise, and F. S. Tautz, New J. Phys. 10, 053012 (2008).

[8] C. Weiss, C. Wagner, R. Temirov, and F. S. Tautz, J. Am. Chem. Soc. 132, 11864 (2010).

[9] C. Weiss, C. Wagner, C. Kleimann, M. Rohlfing, F. S. Tautz, and R. Temirov, Phys. Rev. Lett. 105, 086103 (2010). 
[10] M. Haruta, CATTECH 6, 102 (2002).

[11] L. Barrio, P. Liu, J. A. Rodríguez, J. M. Campos-Martín, and J. L. Fierro, J. Chem. Phys. 125, 164715 (2006).

[12] T. Fujitani, I. Nakamura, T. Akita, M. Okumura, and M. Haruta, Angew. Chem., Int. Ed. 48, 9515 (2009).

[13] P. Jelinek, R. Pérez, J. Ortega, and F. Flores, Phys. Rev. Lett. 96, 046803 (2006).

[14] A. Zanchet, A. Dorta-Urra, O. Roncero , F. Flores, C. Tablero, M. Paniagua, and A. Aguado, Phys. Chem. Chem. Phys. 11, 10122 (2009).

[15] J. Tersoff and D. R. Hamann, Phys. Rev. Lett. 50, 1998 (1983); Phys. Rev. B 31, 805 (1985).

[16] J. M. Blanco, C. González, P. Jelinek, J. Ortega, F. Flores, and R. Pérez, Phys. Rev. B 70, 085405 (2004); J. M. Blanco, F. Flores, and R. Pérez, Prog. Surf. Sci. 81, 403 (2006).

[17] See Supplemental Material at http://link.aps.org/ supplemental/10.1103/PhysRevLett.108.246102 for (i) analysis of different $\mathrm{Au}, \mathrm{W}$, and mixed $\mathrm{Au} / \mathrm{W}$ tips, (ii) other Au-tip $+\mathrm{H}_{2}$ geometries, (iii) spontaneous $\mathrm{H}_{2}$ molecules' dissociation on clean Au-tip (two videos), and (iv) stability of H-sensitized Au-tip on PTCDA/Au(111) interface (video).

[18] J. P. Lewis, P. Jelínek, J. Ortega, A. A. Demkov, D. G. Trabada, B. Haycock, H. Wang, G. B. Adams, J. K.
Tomfohr, E. Abad, H. Wang, and D. A. Drabold, Phys. Status Solidi B 248, 1989 (2011).

[19] J. D. Sau, J. B. Neaton, H. J. Choi, S. G. Louie, and M. L. Cohen, Phys. Rev. Lett. 101, 026804 (2008).

[20] J. I. Martínez, E. Abad, C. González, J. Ortega, and F. Flores, Org. Electron. 13, 399 (2012).

[21] J. I. Martínez, E. Abad, F. Flores, J. Ortega, and G. Brocks, Chem. Phys. 390, 14 (2011).

[22] N. Koch, Chem. Phys. Chem. 8, 1438 (2007); N. Dori, M. Menon, L. Kilian, M. Sokolowski, L. Kronik, and E. Umbach, Phys. Rev. B 73, 195208 (2006).

[23] S. Baroni, A. D. Corso, S. de Gironcoli, and P. Giannozzi, QUANTUM-ESPRESSO, http://www.quantum-espresso.org (2005).

[24] We have used the PBE0 exchange-correlation functional, and have also taken into account the $\mathrm{H}$ zero-point energy (0.27 eV for the $\mathrm{H}_{2}$ molecule and $0.07 \mathrm{eV}$ for $\mathrm{H}$ on $\mathrm{Au}$ [25]).

[25] F. Abild-Pedersen, J. Greeley, F. Studt, J. Rossmeisl, T. R. Munter, P. G. Moses, E. Skulason, T. Bligaard, and J. K. Nørskov, Phys. Rev. Lett. 99, 016105 (2007).

[26] R. E. Walkup, D. M. Newns, and P. Avouris, Phys. Rev. B 48, 1858 (1993).

[27] S. Gao, M. Persson, and B. I. Lundqvist, Phys. Rev. B 55, 4825 (1997). 